\title{
The Prospects of Using Fuzzy Approaches to Ecological Risk Assessment
}

\author{
Oleg Uzhga-Rebrov, Rezekne Higher Education Institution, \\ Galina Kuleshova, Riga Technical University
}

\begin{abstract}
The issue of environmental quality improvement has been receiving much attention in the developed countries in recent years. Due to that, the role of assessment of ecological risks associated both with natural events and technogene activity of humans is increasing. Previous approaches to the assessment of ecological risks were fully based on statistical data and expert evaluation of potential losses and probabilities of unfavourable consequences. When this kind of assessment is carried out, it is assumed explicitly that experts are able to evaluate point probabilities. However, such assumptions are far from being true. As a result, fuzzy approaches to ecological risk assessment became popular lately. This paper focuses on two practical approaches of that kind. The paper is aimed at attracting practical attention to new up-to-date techniques that could be successfully applied to assess ecological risks in Latvia.
\end{abstract}

Keywords -linguistic categories, safety analysis, fuzzy numbers, fuzzy representation of knowledge, fuzzy rules.

\section{INTRODUCTION}

A great deal of attention has been paid lately to the problem of environment protection in the developed countries. The quality of environment may be negatively affected by several nature factors: earthquakes, volcano eruption, hurricanes, floods, draught, etc. These factors have existed during the whole history of the Earth evolution, so ecosystems have sufficient and strong enough protection mechanisms to resist to the action of different unfavourable nature factors. Even greater negative impact on the environment is made by technogene human activity. All the negative effects associated with different kinds of such activity can be divided into two large groups: (1) irrational use of the existing nature resources, which might cause large problems for further generations (externalities between the generations) and (2) different kinds of environment pollution occurring as a result of economic activity. The necessity of avoiding or lessening negative effects of the second group has been recognised long ago and important measures are undertaken to reach the goals stated. The actions aimed at lessening these effects are planned and accomplished on the basis of the respective measurements and monitoring of relevant factors. This kind of approach can be quite validated when dealing with already existing deviations in the functioning of ecosystems that can be evaluated directly but real causes of these deviations can be discovered and proper corrective actions can be developed. It is more difficult to assess ecological risks. The problem is that the risks are related to the potential negative effects on the environment which might occur due to different natural and technogene reasons. For example, what consequences might follow potential long-term period of the draught in the region or potential accident at a chemical enterprise? In such situations it is necessary to evaluate possible losses at different levels of the negative impact and chances of occurrence of such losses. During a long period, the chances of occurrence of different kind of losses were commonly evaluated by probabilities assuming that all possible levels of losses constitute a complete group of random events. Thus, the assessment of ecological risks within standard approach includes two dimensions: possible losses and probabilities of occurrence of losses at different levels of possible influence.

A question then arises: how to obtain the necessary initial data for evaluating ecological risks? If sufficient statistical material is available, the task is not difficult. A visual example can be the assessment of fire risk in a building of a certain type. Rich statistical material allows one to justly evaluate the relative frequency of fire occurrence in this kind of buildings. This frequency can be correctly assumed as the probability of fire occurrence in a certain building. Estimated losses caused by the fire can be easily calculated as average losses related to previous fires. Based on the obtained evaluations, the risk can be easily evaluated and the cost of insurance police can be determined.

Unfortunately, the presence of sufficient statistical material in the assessment of ecological risks is the exception rather than the rule. The uniqueness of such situations and their rear occurrence do not allow one to use efficient apparatus of statistics to obtain necessary evaluations. To cope with the lack of objective information, expert evaluation is frequently used. Experts-specialists based on their professional knowledge, experience and, sometimes, intuition can provide the necessary data. However, the problem of confidence degree evaluation for the data obtained appears. Practically, there are no suitable methods for solving that task if objective initial information is completely unavailable; though methods exist that 
allow one to a priori evaluate potential nonobjectivities of the expert in the planned evaluation but these techniques might provide only little help as regards the confidence of evaluations of the particular expert, whereas the use of group expert evaluation may only make the task even more complicated in the case when the evaluations of separate experts turn to be sufficiently contradictive.

A lot of techniques are developed for obtaining and using uncertain probabilistic evaluations; these include interval probabilities, second order probabilities etc. The shortcomings of these techniques are their complexity and poor interpretability of the uncertain results obtained.

In 1965, L.Zadeh [6] proposed a principally new conceptual basis for dealing with vague, imprecise information - fuzzy set theory. The theory was widely developed during the past years. Nowadays, fuzziness is used practically in all fields of scientific and practical activity. This paper considers two practical examples of using fuzzy sets and fuzzy rules in ecological risk assessment. Based on the analysis of the considered examples some generalized conclusions are drawn and recommendations on applying fuzzy techniques for ecological risk assessment are given.

\section{BASIC CONCEPTS OF FUZZY SET THEORY}

Nowadays there are a large number of textbooks and reference guides available on fuzzy set theory. As an example of a textbook, we can mention [1]; while a more detailed and thorough description of the theory can be found in [3].

Fuzzy set theory has been developed to cope with vague and imprecise categories having no sharp boarders. As an example let us consider the variable Age of individuals. Let us assume that the value of that variable may be within the interval $[0,100]$ years. Let us distinguish separate categories in that interval, say, children, young people, middle age people and older people. These categories can be distinguished by fixing boundaries between separate categories. Let us assume that the value of boarder between young people and middle aged people is set as 35 years. Then a person at the age of 34 years 11.5 months will be ascribed to the category of young people but a person at the age of 35 years 0.5 months will be ascribed to the category of middle aged people. A slight difference in the age makes one to ascribe these persons to different age categories, which is not justified. Now, let us assume that the age of an individual is determined approximately. According to the above-mentioned age classification, this individual can be ascribed to a single category only. But what can be done if, based on his look, it is possible to ascribe him both to the category of young people and the category of middle-aged people? A great deal of similar examples can be offered: classification of individuals according to weight, classification of temperature etc.
To ensure a more flexible and convenient operation of different kind of classification categories, the boundaries between the categories can be somehow made vague. This idea is implemented in the concept of linguistic category (alternatively, linguistic term). Different values of relevant variable may belong to a certain linguistic category with different extent (strength). Moreover, some values of the variable may belong to different linguistic categories with equal or different extents.

Let us consider a case study. Assume that as a result of accident, a harmful substance has entered the environment, which might cause unfavourable effects of different extent. It is clear that the degree of unfavourable influence will depend on the concentration of that substance in the external environment. Let us distinguish these fuzzy linguistic categories of the degree of the unfavourable effect at the scale of harmful substance concentration: low, medium and high. These categories are conditionally represented as membership function graphs in Fig. 1.

As can be seen from Fig. 1, at the concentration values less than $c_{1}$, the extent of pollution will definitely be low. At the values of concentration $\mathrm{c}_{1} \leq$ $\mathrm{C} \leq \mathrm{c}_{4}$ the extent of membership of pollution to the fuzzy category low decreases in succession from 1 to 0 . At the values of concentration $\mathrm{c}_{2} \leq \mathrm{C} \leq \mathrm{c}_{5}$, the extent of pollution membership in a fuzzy category medium increases in succession from 0 to 1 . At the values of concentration $\mathrm{c}_{2} \leq \mathrm{C} \leq \mathrm{c}_{4}$ actual pollution can also be ascribed to both fuzzy category low and fuzzy category medium with different degrees of membership. At the concentration equal to $c_{3}$, the degrees of membership in both categories will be similar. Degrees of membership to other fuzzy categories can be interpreted in the same way.

In practical applications, fuzzy numbers are of great importance. A fuzzy number is a fuzzy subset defined in a set of real numbers $\mathbf{R}$. As an example, two triangular fuzzy numbers $A_{1}$ and $A_{2}$ are shown in Fig.2. These numbers have received their name because of the form of membership function graphs. There also exist other types of fuzzy numbers. Arithmetic operations on fuzzy numbers can be performed according to the rules of fuzzy arithmetic.

A set of interconnected variables describing a certain set of the real world is often called a system. If the states of variable values are expressed by means of fuzzy sets and/or other fuzzy operators, this kind of system is called a fuzzy system. In the most general case, fuzzy systems can be classified as model-based, knowledge-based and also hybrid [2]. Fuzzy systems that are based on knowledge are the result of traditional modelling of systems; they employ appropriate fields of fuzzy mathematics (fuzzy analysis, fuzzy mathematic operations, fuzzy relations etc.). In knowledge-based fuzzy systems, the correlations among variables are described by means of a set of fuzzy rules. 


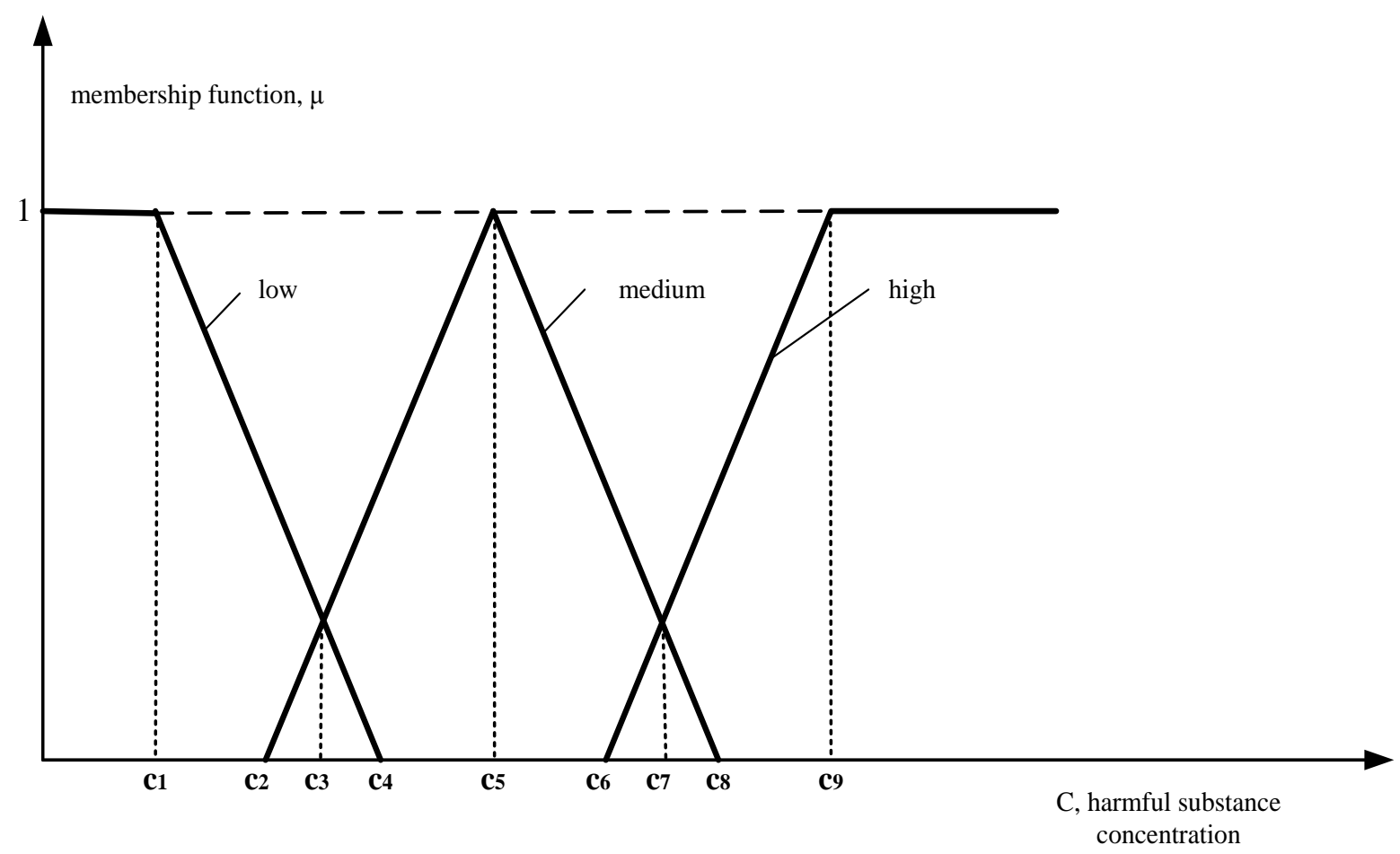

Fig.1. Graphs of membership functions of fuzzy categories of the degree of unfavourable impact on the environment at the scale of harmful substance concentration

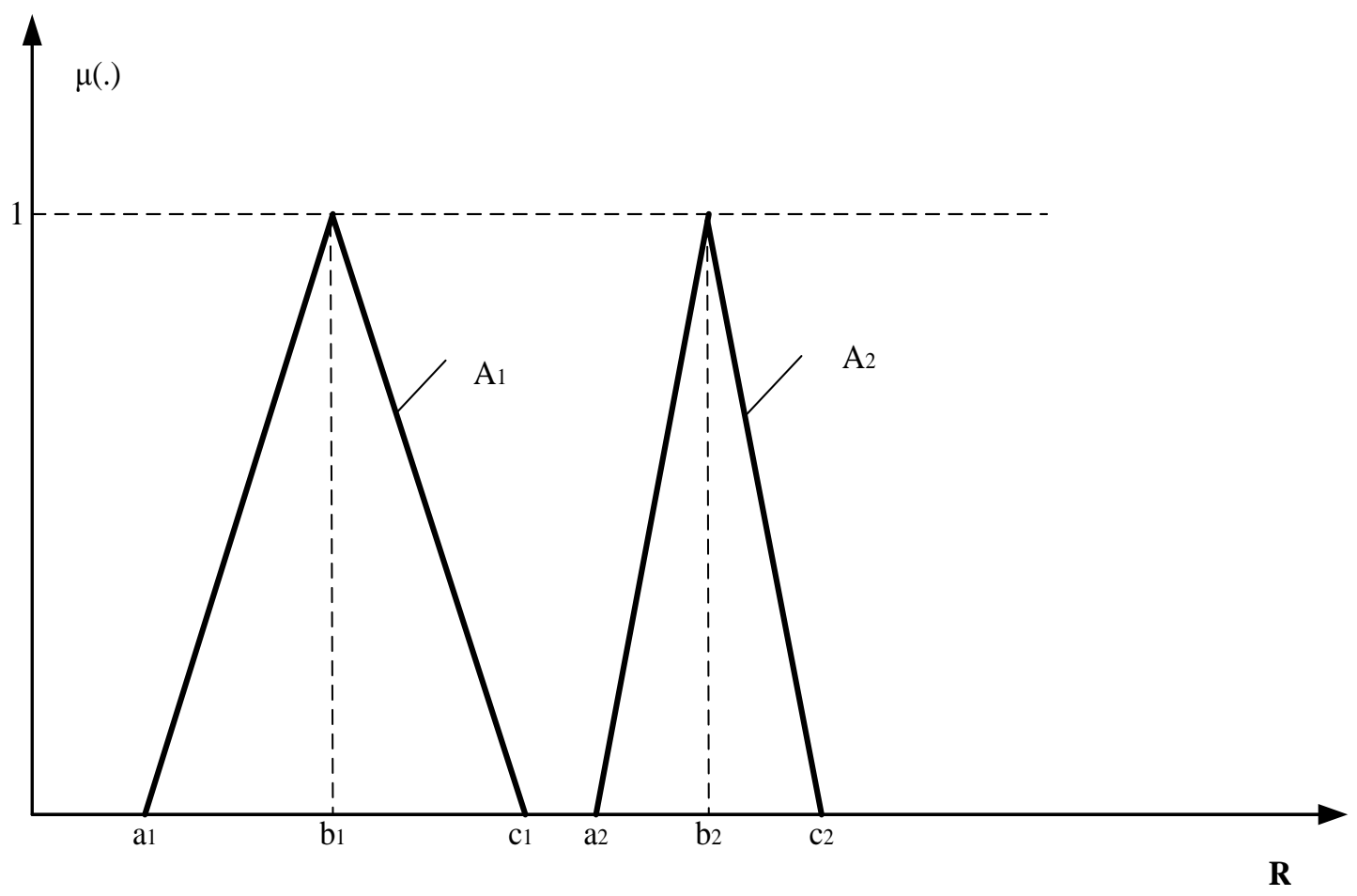

Fig.2. Sample representation of triangular fuzzy numbers

These rules are formed using experts' opinions and evaluations that represent their knowledge in the subject area.
This knowledge can be initially expressed in the terms of natural language and then formally transformed into the fuzzy forms. 


\section{AN EXAMPLE OF TECHNICAL RISK ASSESSMENT USING A FUZZY MODEL-BASED SYSTEM}

To illustrate the above-mentioned technique, let us use the data shown in [5]. The paper provides a detailed analysis of fuzzy evaluation of technical system safety. It is clear that ecological risk is inversely proportional to the safety of relevant technical system activity. The matter is that the lower the safety of, say, equipment for manufacturing dangerous chemical substance, the higher the probability of its breakage and the higher the risk that during the accident the environment will heavily suffer. Thus, the assessment of technical risks is actually equivalent to the assessment of ecological risks.

The event of technical system breaking or degradation is a fundamental element of safety analysis, and the main problem is how to model damage events with complicated possible states. The qualitative analysis of safety has to assess not the chances of real damage of system element, but the chances of occurring such element state at which the element is still functioning but its parameters have changed in such way that the chance of damage is growing. In this sense, the discussion is about different kinds of damage.

Standard statistical approach to the assessment of technical system safety suffers from a shortcoming that quite frequently initial data are not sufficient to induce reliable evaluations. The fuzzy set-based approach helps to take into consideration the insufficiency of the initial data. In the considered variant of the approach, it is assumed that relevant initial data are represented as triangular fuzzy numbers and operations on them are carried out by the rules of fuzzy arithmetic.

Technical system safety analysis can be performed in two variants [5]: (1) in the form of structure function synthesis and (2) in the form of damage tree analysis. The first variant is used at the stage of system development but the second one - at the stage of system exploitation. In any case, the major factors of system safety are fuzzy reliability of normal functioning of the i-th component, $\widetilde{\operatorname{Re}} l(i)$, fuzzy average number of damages of the $i$-th component at time unit $\tilde{\lambda}(\mathrm{i})$ and fuzzy average time between damages of the $\mathrm{i}$-th component $1 / \widetilde{\lambda}(i)$.

In parallel fragments of the system, components are included in parallel; one of the components is in working state while the other components are in the standby state. When the functioning component is out of order, the next component is turned on and so on. Thus, a complete abandoning of the fragment will only occur when all its components are damaged.

Fuzzy likelihood of the normal functioning of the fragment at time moment $t$ is calculated by this expression:
$\tilde{\operatorname{Re}} l(f r)=\prod_{i=1}^{n} \tilde{\operatorname{Re}} l(i)=\coprod_{i=1}^{n} e^{-\tilde{\lambda}(i) t}=e^{-n \tilde{\lambda} t}$

where $\mathrm{n}$ - number of the components that are connected in parallel;

$\tilde{\lambda}$ - average number of damages of the component.

In [5] it is shown that in the case of $n$ in parallel connected components with constant value $\tilde{\lambda}$, that are represented in the form of triangular fuzzy numbers (a, b, c) (see Fig.2) the resulting triangular number for $\tilde{\operatorname{Re}} l(f r)$ in expression (1) can be represented as

$$
\tilde{\operatorname{Re}} l(f r)=\left(e^{-a n}, b^{-b n}, c^{-c n}\right)
$$

This kind of approximation brings a small error but significantly simplifies the calculations.

For a fragment of technical system with $n$ connected in sequence elements we have

$$
\tilde{\lambda}(f r)=\sum_{i=1}^{n} \tilde{\lambda}(i)
$$

If the values of $\tilde{\lambda}(i)$ are represented in the form of triangular fưzzy numbers $\left(a_{i}, b_{i}, c_{i}\right)$, the calculation of the value $\tilde{\lambda}(f r)$ is reduced to a simple addition of these numbers according to the rules of fuzzy arithmetic.

After the value of $\tilde{\lambda}(f r)$ is calculated, it is possible to calculate in sequence the value of $\lambda$ (system) for the existing connections of the fragments.

What is the advantage of the considered fuzzy approach to the calculation of technical system safety as compared to the standard probabilistic approach? The probabilistic approach foresees point evaluations of the probabilities of fuzzy events - damages of system's components. Under the conditions of the shortage of the initial information, the confidence of the obtained evaluations can be extremely low. In the considered approach, the incompleteness and insufficiency of the initial information is explicitly modelled by means of triangular fuzzy numbers. It is natural that the fuzziness of the initial evaluations is translated into the fuzziness of the result. However this kind of fuzziness is better than point evaluations with unknown level of confidence. At least, under the fuzzy results it can be clearly seen in which limits and with which confidence the real value of the factor under evaluation can be expected. To lessen the degree of imprecision of the results, the worst values of the evaluations can be used as a basis and conclusions can be drawn on the basis of such pessimistic evaluation. Besides, defuzzification techniques can be used that enable making more narrow intervals of fuzzy resulting evaluations. 


\section{AN EXAMPLE OF TECHNICAL RISK ASSESSMENT USING A FUZZY KNOWLEDGE-BASED SYSTEM}

In [4], the authors describe a technique for evaluating the ecological risk that is connected to mercury emissions when mining gold in Canada. The level of mercury in sediments, water and biota is the basis for making political and technical decisions on making correcting procedures. It is clear that the chemical analysis is the major source of information for the evaluation of potential harmful impact on the environment. To obtain data reliable by many points of evaluation, large financial resources and numerous educated staff is required. The authors point out that when similar task was solved in Sweden, mercury content was evaluated in 1836 lakes. However, when forecasting the development of situation evolution in the future at so numerous initial data, prediction error was within $50 \%$. This gives evidence that to receive reliable results, even larger amount of initial data is required.

Based on the analysis of the state of the art, the authors [4] have suggested a simple heuristic approach to the evaluation of ecological risks in the area of gold mining. The evaluation of mercury content in the water, sediments and live organisms is made using terms of linguistic categories high, medium and low. For all relevant factors of the task, the identification of linguistic categories and construction of membership function graphs is made. In their work the authors treat the values of membership functions as the Degree of Belief (DoB).

Sediments are both carriers and sources of contaminants in aquatic systems. The possibility of $\mathrm{Hg}$ bioaccumulation is influenced by the contamination level of sediment. The Index of Geoaccumulation $\left(\mathrm{I}_{\mathrm{geo}}\right)$ as a quantitative measure of metal pollution in aquatic sediments [4] uses the relationship between concentration $\mathrm{C}$ of the element in the sediment and background in fossil argillaceous sediment (B):

$$
I_{\text {geo }}=\frac{\log _{2} C}{1,5 B}
$$

For making a fuzzy evaluation of the extent of presence of $\mathrm{Hg}$ in sediments, the authors have classified these types of sediments:

Type 1: Comprises gravels, white or light grey clay or sand, limestone, sandstones;

Type 2: comprises any reddish clayey or sand sediment;

Type3: comprises organic-rich sediments.

Using the knowledge and experience of experts, a set of rules connecting the type of sediments and fuzzy values of other relevant factors with the fuzzy amount of hydrargyrum at the point of measurement, is constructed. All the rules are shaped as an expert system $\mathrm{HgEx}$. As the authors point, "HgEx is heuristic system, which accommodates imprecise data input for many variables, such as $\mathrm{pH}$, Eh, water conductivity, biomass productivity, water transparency and contamination factor. The system does not replace the monitoring programs because all data are obtained in field trip, but rather reduces the need for accurate results to provide a preliminary but conclusive risk assessment report".

\section{RESULTS AND CONCLUSIONS}

This paper considered two practical approaches to the fuzzy evaluation of ecological risks. One of the approaches is based on fuzzy models, while the other one employs a fuzzy representation of knowledge. Fuzzy evaluation of technical risks has already found practical application. Its advantages are in the opportunity to use vague, imprecise initial Information, which is not possible when using standard statistical approach. In no case it means the rejection to use this kind of approach. In the case, when sufficient statistical data are available (e.g. in case of fires or aviation accidents), the statistical approach has undoubted advantages. However, in practical tasks of ecological risks evaluation, the lack of sufficient initial data is the rule rather than the exception; so here fuzzy techniques are preferable.

As shown in Section 3, fuzzy evaluation of technical risks requires only fuzzy evaluations of initial factors. By regulating the basis of relevant triangular fuzzy numbers, practically any degree of non-knowledge regarding the factors to be evaluated can be modelled. It is natural that the fuzzier the initial data are, the fuzzier the results will be. However, even at the sufficient fuzziness of the results, practically there is no alternative to the fuzzy approach. In this kind of state, it is simply impossible to get more or less reliable probabilistic evaluations.

The practical method for the assessment of technical risks presented in Section 3, can be successfully employed in numerous other situations. A logically validated requirement for such application is a tight functional relation between the initial data and the resulting evaluations.

Another practical approach to assessing ecological risks that is discussed in Section 4 is completely based on the use of the knowledge and experience of experts. Different versions of that technique might also find a wide application in other fields where it is possible to determine heuristic dependencies between the initial data and the resulting evaluations. Fuzzy rules are excellent tool for expressing relevant dependencies.

Other conceptual approaches to the assessment of ecological risks at the imprecise initial information also exist; one of such approaches is based on the theory of possibilities. The consideration of alternative approaches is, however, beyond the scope of this paper, first due to the limited paper size and second due to numerous advantages of fuzzy techniques. 
Uzhga-Rebrov O., Kuleshova G. THE PROSPECTS OF USING FUZZY APPROACHES TO ECOLOGICAL RISK ASSESSMENT

\section{REFERENCES}

[1] M. Ganesh, Introduction to Fuzzy Sets and Fuzzy Logic. PHI Learning Pvt. Ltd, 2006, 256 p.

[2] G.J. Klir, Uncertainty and Information. Foundation of Generalized Information Theory. John Wiley\&Sons, Inc., 2004.

[3] G.J. Klir and Yuan Bo, Fuzzy Sets and Fuzzy Logic. Prentice Hall, 1995, $592 \mathrm{p}$.

[4] M.M. Veiga and J.A. Meech, "Application of Fuzzy Logic to Environmental Risk Assessment", Department of Mining and
Mineral Process Engineering, University of British Columbia, Vancouver, Canada, http://www.mining.ubc.ca/ faculty/meech/fuzchile.htm.

[5] L. Warren, "On Modeling Hybrid Uncertainty in Information", Australian Department of Defense. http://www.dtic.mil/cgibin/GetTRDoc?AD=ADa467752.

[6] L. Zadeh, "Fuzzy Sets", Information Control, 1965, 8, pp. 338 -353 . 